\title{
Dislocation Cross-Slip in Nanocrystalline fcc Metals
}

\author{
E. Bitzek, C. Brandl, P. M. Derlet, and H. Van Swygenhoven \\ Materials Science \& Simulation, ASQ/NUM, Paul Scherrer Insitut, CH-5232 Villigen PSI, Switzerland
}

(Received 21 February 2008; published 11 June 2008)

\begin{abstract}
Constant strain rate molecular dynamics simulations of nanocrystalline Al demonstrate that a significant amount of dislocations that have nucleated at the grain boundaries, exhibit cross-slip via the Fleischer mechanism as they propagate through the grain. The grain boundary structure is found to strongly influence when and where cross-slip occurs, allowing the dislocation to avoid local stress concentrations that otherwise can act as strong pinning sites for dislocation propagation.
\end{abstract}

DOI: 10.1103/PhysRevLett.100.235501

PACS numbers: 62.25. $-\mathrm{g}$, 47.11.Mn, 61.72.Lk

Cross-slip, in which screw dislocations leave their habit planes and propagate to another glide plane [1,2], plays a key role in plasticity. Such a process can decrease internal stress fields exerted, for example, by dislocation pile-ups, resulting in the formation of dislocation cell structures [3]. Macroscopically cross-slip reduces the strain hardening rate with increasing strain, which in bulk single crystals is associated with stage III hardening [4]. In nanocrystalline metals, dislocation pile-up formations are however suppressed due to the spatial restrictions associated with the small grain size. Molecular dynamics simulations performed on nanocrystalline structures have demonstrated that in the absence of defects within the grain interior in nanocrystalline metals, grain boundaries (GBs) can act as both sources and sinks for partial and perfect dislocations [5-13]. During propagation, dislocations can become temporarily pinned at GB ledge structures and their associated stress intensities, and upon depinning they propagate further on the slip plane in which the dislocation had originally nucleated [10]. This picture is supported by in situ $\mathrm{x}$-ray diffraction experiments that can probe the dynamics of plasticity, demonstrating that no permanent dislocation network is accumulated during plastic deformation [14,15].

In this Letter it is shown that dislocation cross-slip plays an important role in the plastic deformation of fully threedimensional $\mathrm{Al}$ nanocrystalline samples. In particular, it is demonstrated that cross-slip provides a mechanism for the propagation of a perfect dislocation to circumvent stress intensities in the GBs, allowing the dislocation to deposit itself in more favorable GB regions such as triple lines, and GB regions under tensile stress. This observation can explain the lack of an increase in the flow stress with increasing strain observed in atomistic simulations of bulk nanocrystalline metals.

For the present work, a nanocrystalline sample containing 100 grains characterized by a mean grain diameter of $11.5 \mathrm{~nm}$ was constructed using the Voronoi tessellation procedure [16]. The details of the preparation of such fully three-dimensional GB networks can be found elsewhere [17]. The sample is strained uniaxially with a constant strain rate and the Poisson contraction in the lateral directions is modeled by the standard Parrinello-Rahman baro- stat [18], an algorithm allowing the size of the simulation cell to vary in response to the external stress conditions. To model the interatomic interactions between the $\mathrm{Al}$ atoms, the $\mathrm{Al}$ embedded-atom empirical potential of Mishin et al. [19] is used. This interatomic potential was chosen because it is known to result in the nucleation of the leading and trailing partial dislocations at GBs, resulting in the propagation of a perfect dislocation within the timescale of the molecular dynamics simulation [20]. For analysis at the atomic level, the medium range order analysis procedure [21] is used in which atoms are coloured according to their local symmetry where gray represents face centered cubic (fcc) atoms, red hexagonal closed packed (hcp) atoms, green other 12-coordinated atoms and blue non-12coordinated atoms. Stress distributions in the GB are visualized using the local hydrostatic pressure calculation according to Cormier [22]. For both atomistic visualization methods, the atomic quantities and corresponding positions are averaged over $250 \mathrm{fsec}$ to minimize the effect of thermal noise.

Figure 1 (left axis) displays the resulting stress-strain curve for the chosen strain rate of $1 \times 10^{8} / \mathrm{sec}$. Such a high strain rate is typical for molecular dynamics simulations and results in the observed plasticity mechanisms being predominantly stress driven with some thermal assistance. However in experiments, which are performed at significantly lower strain rates, the situation can be quite the opposite with the rate limiting plasticity mechanism often being characterized as thermally driven with some stress assistance. In Fig. 1 an extended elastic-plastic transition region is seen until a flow stress of approximately 1.5 GPa. Beyond this regime the flow stress decreases with increasing plastic strain. From inspection of slip traces derived from a slip vector analysis [23] and detailed atomic inspection, the dislocation activity was enumerated and is shown in Fig. 1 (right axis) as the cumulative number of slipped dislocations as a function of strain. Significant slip activity (in which dislocations traverse grains) starts at approximately $4 \%$ strain, just before the maximum flow stress is reached, a result that correlates well with a recent experimental investigation [24]. The irreversible elasticplastic transition region occurring below $4 \%$ strain is pre- 


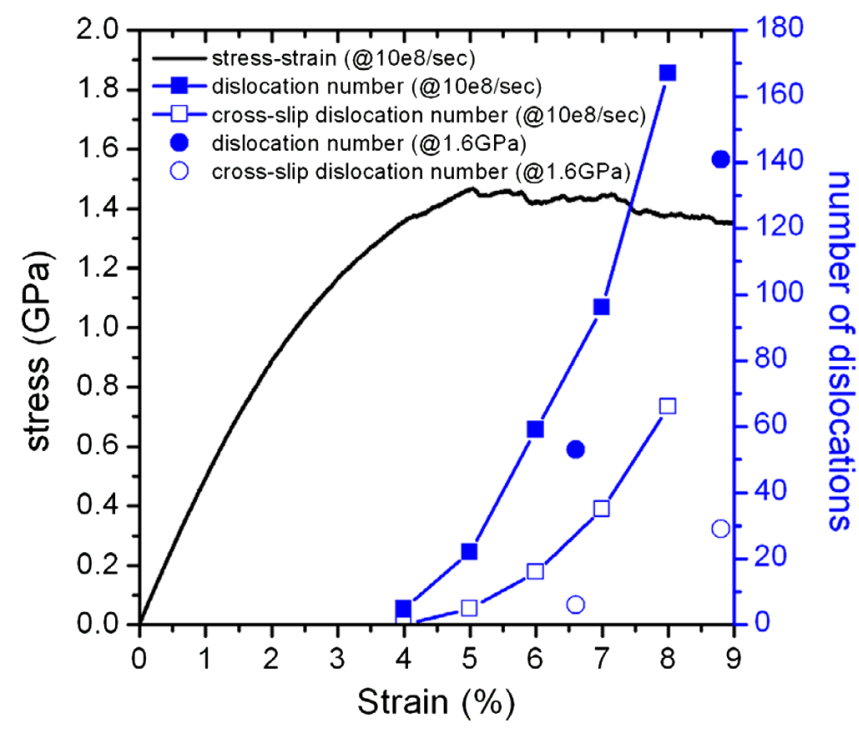

FIG. 1 (color online). (Left axis) Stress-strain curve for a constant strain rate of $1 \times 10^{8} / \mathrm{sec}$. (Right axis) Total cumulative number of dislocations and number of these dislocations that have cross-slipped as a function of strain for the constant strain rate simulation and constant load simulation performed at a uniaxial tensile stress of $1.6 \mathrm{GPa}$.

dominantly characterized by dislocation nucleation and other GB mediated processes. Also shown in Fig. 1 is the number of these dislocations that cross-slip, demonstrating a slight increase in percentage of cross-slipped dislocations with increasing strain. An in-depth analysis of all dislocation activity and its relation to local stress conditions, GB structure and GB network structure will be published elsewhere [25]. In what follows, we present two examples of dislocations that undergo cross-slip, paying particular attention to the important role of the surrounding GB structure. To aid visualization of these examples, movies are provided in the auxiliary material for this letter [26].

Figure 2 displays a region where a leading and trailing partial dislocation have nucleated at a GB, propagated and cross-slipped through the grain, and eventually been absorbed in the surrounding GB network. Some of the grains that surround the central grain (grain 65) in which the cross-slipping dislocation exists, are visualized as gray volumetric regions. The central grain is not shown apart from the partial dislocation cores and the stacking fault in between (indicated by non-fcc atoms colored according to their medium range order classification) just after the leading partial dislocation has nucleated at GB 65-59 (indicated by the red arrow), and at a time at which the dislocation has already cross-slipped a number of times. We note that the initial leading partial dislocation segment fluctuated in size for approximately 35 psecs before the nucleation of the trailing partial and propagation of the resulting perfect dislocation into the grain. The slip systems populated by the dislocation as it cross-slipped through the grain are indicated as transparent yellow surfaces that are bound by their intersection with the surrounding GBs and the
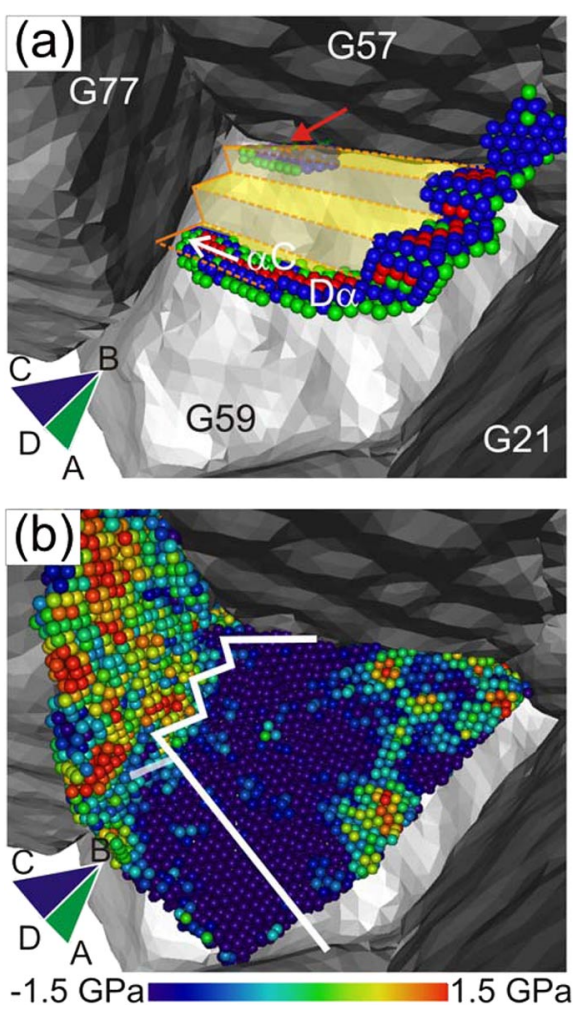

FIG. 2 (color online). (a) Snapshot of a dislocation that during propagation has cross-slipped several times between slip planes (a) and (b) schematically indicated by the shaded yellow planes. Only the non-fcc atoms within the central grain are shown to visualize the dissociated core of the perfect dislocation using the medium range order classification scheme. (b) Non-fcc atoms of GBs 65-77 and 65-59 onto which the dislocation deposits itself. The viewing angle is similar to (a) and the atoms are colored according to their local hydrostatic pressure as indicated by the associated linear color bar, where the white line represents the region onto which the dislocation is deposited.

instantaneous position of the dislocation core. Also shown is the Thompson tetrahedron [1] that indicates the relevant slip systems and the Burgers vector, $D C$, as a white arrow. Initially a leading and then trailing partial dislocation nucleates on the (a) plane and approximately 1 psec after nucleation of the trailing partial, the screw segments of the perfect dislocation has cross-slipped onto the (b) plane. Over the next 10 psecs the screw segment of the dislocation cross-slips alternatively onto planes (a) and (b) as it propagates through the entire grain. This leaves behind a stair case of edge segments bound by stair rod dislocations [27] which propagate to the right until they disappear in the surrounding GB structure (mostly GB 65-21).

Detailed atomic inspection of the dislocation as a function of time revealed that cross-slip occurs by the leading partial dislocation first changing its glide plane by the creation of a stair rod dislocation [27] with which the trailing partial reacts thereby also changing its glide plane. Such a process of cross-slip falls under a class of mechanisms commonly referred to as the Fleischer mechanism $[1,28]$. 
When and where cross-slip occurs may be rationalized by inspection of Fig. 2(b), which has the same orientation as Fig. 2(a). In Fig. 2(b) the GB atoms of GBs 65-77 and 65-59 are displayed and colored according to their local hydrostatic pressure before deposition of the dislocation in which blue represents atoms in tension and red in compression. The region in which the dislocation segment is deposited is indicated by a white line. What becomes evident is that through cross-slip the screw component of the dislocation chooses a path that avoids the compressive regions of GB 65-77, allowing the dislocation to deposit its predominantly edge component into the regions of GB 6559 that are under tension. Moreover, it is found that crossslip is always initiated at the intersection of the GB with the dislocation, or very close to this region, indicating that the strong variations in hydrostatic pressure within the GB play the crucial role in providing the complex stress state necessary for cross-slip to occur. Closer temporal analysis of the dynamics to cross-slip also revealed that attempts to cross-slip can fail. An example of this is shown schematically in Fig. 2(b), where the transparent white line indicates a cross-slip event that retracted after approximately one psec in favor of continued propagation along the slip plane indicated by the full white line.

Figure 3 shows another example of a perfect dislocation undergoing cross-slip, now in grain 86. A similar visualization scheme is used as in Fig. 2. Through Figs. 3(a)-3(c) we show in more detail the temporal sequence of the dislocation as it cross-slips through the grain. For the same orientation as these figures, Fig. 3(d) displays the non-fcc atoms shaded according to their local hydrostatic pressure for GB 86-7 and GB 86-80 at a time before deposition of the dislocation. Inspection of Fig. 3 demonstrates once again the crucial role of the surrounding GB
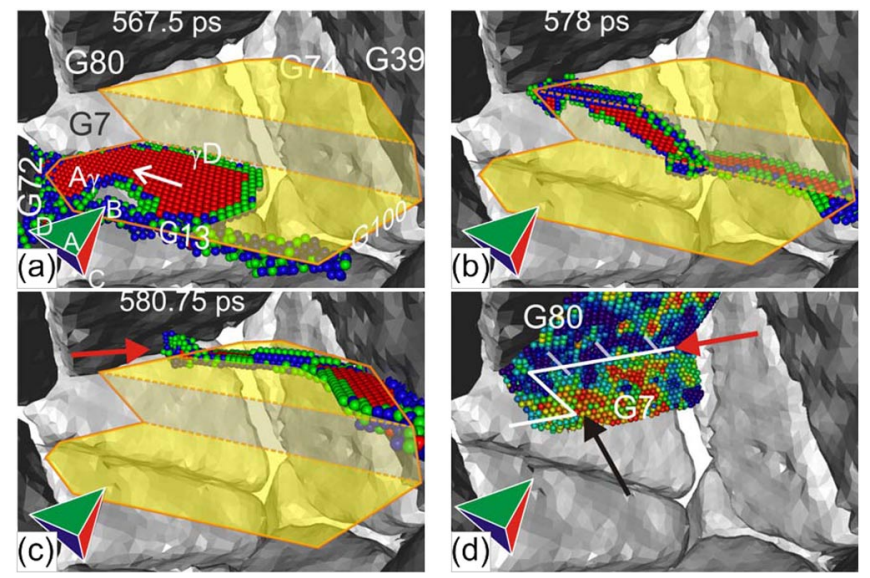

FIG. 3 (color online). (a)-(c) Three snapshots of a perfect dislocation that cross-slips twice to avoid a compressive stress region of the neighboring GB 86-7 shown in (d). The visualization method is similar to that described in Fig. 2. In all figures, grains 13 and 100 are not shown, and in (a) the triple junction line at which the perfect dislocation nucleates is displayed as the associated non-fcc atoms. structure in determining the final path of the cross-slipping dislocation. The leading partial dislocation nucleates at the triple junction, GB 86-13-72, whereas the trailing partial nucleates in GB 86-13 resulting in a perfect dislocation with Burgers vector $A D$ [see Fig. 3(a) where the disassociated dislocation and its corresponding stacking fault are evident]. This dislocation propagates in plane (c) until one of the end segments of the leading partial dislocation encounters a GB region exhibiting strong local compressive hydrostatic pressures [indicated by the black arrow in Fig. 3(d)]. Here, in this region of the GB, the dislocation cross-slips onto plane (b). During subsequent propagation [Figs. 3(b) and 3(c)], the edge segment propagates to the right and the screw segment double cross-slips back to the (c) plane resulting in an evolving stair rod structure [Fig. 3(c)]. Cross-slip therefore allows for the dislocation to avoid the compressive regions of GB 86-7 with the left segment of the GB (now on a different (c) plane) depositing itself along a triple line under tension [indicated by the red arrow in Fig. 3(d)]. Before complete absorption of the remaining dislocation structure on the (c) plane, the dislocation is seen to make three attempts to cross-slip onto the (b) plane as schematically indicated by the transparent lines in Fig. 3(d), an example of which is seen in the red arrowed region of Fig. 3(c). The lifetime of this dislocation is particularly revealing since it provides a clear example of a dislocation that is pinned to a region of GB (GB 86-7), indicated by the back arrow in Fig. 3(d), for approximately 1 psec before depinning via the cross-slip mechanismthat is, the dislocation is able to pass the pinning region by double cross-slipping to a parallel slip plane so that it can continue to propagate through the grain. Close inspection of the pinning region reveals that the strong variations in the hydrostatic pressure of this GB region [Fig. 3(d)] are due to misfit structures accommodating the missorientation of grains 86 and 7 .

Dislocation cross-slip is seldom seen in molecular dynamics simulations of single crystal plasticity [29], except when specifically addressed in specially designed molecular dynamics studies [30-32]. The present work provides a first molecular dynamics example of cross-slip occurring in a plastically deforming three-dimensional GB network. The reason that such activity has until now not been reported in such nanocrystalline structures $[7,8,10,12,13,20,33-37]$ may be found in one or more of the following reasons: (i) Past simulations only investigated the onset of dislocation activity. Earlier work on nanocrystalline $\mathrm{Al}$ [10], done using constant stress loading conditions and the embedded-atom method potential of Mishin and Farkas [19], involved detailed atomic analysis only to strains less than 5\%. Figure 1 shows that when continuing such constant stress simulations up to $8.8 \%$ strain, cross-slip is also observed. (ii) Past simulations where performed under constant stress loading conditions. Figure 1 shows that cross-slip occurs at a reduced level for constant load simulations compared to the constant strain rate simulations. (iii) Past simulations were per- 
formed at higher strain rates [10-12,34,37,38]. It is known that in molecular dynamics simulations, the application of higher strain rates result in higher flow stresses [13] thus enabling dislocations to propagate athermally through the stress intensities of the GB as they traverse the grain, i.e., avoiding the possibility to cross-slip. The reduced level of cross-slip observed in the constant load simulation at 1.6 GPa (Fig. 1) have associated strain rates that are approximately 1 order of magnitude higher compared to the constant strain rate simulations of $1 \times 10^{8} / \mathrm{sec}$. (4) The majority of past simulations were performed for fcc metals for which the interatomic potential did not allow the observation of perfect dislocations.

The occurrence of cross-slip, which is classically known to reduce the strain hardening rate resulting from the dislocation pile-up mechanism also explains why in nanocrystalline metals no hardening in simulations is observed once the flow stress is reached. Former molecular dynamics simulations have suggested that dislocation nucleation, propagation and absorption change the GB structure. Some of the stress intensities are relieved upon dislocation nucleation $[10,34,39]$, but many new stress intensities are built up because of the difficulty in relaxing the GB structure after dislocation absorption within the time frame of the simulation $[40,41]$. Inspection of the stress-strain curve in Fig. 1 demonstrates that the accumulation of such stress intensities with increasing strain does not lead to an increase in the flow stress with increasing strain, which can be expected when dislocations are able to avoid these regions via cross-slip.

In summary, constant uniaxial strain rate molecular dynamics simulations of nanocrystalline $\mathrm{Al}$ have shown that a significant amount of dislocations that have nucleated at the grain boundary, exhibit cross-slip via the Fleischer mechanism as they propagate through the grain. Cross-slip is found to be initiated by the stress signature of the local grain boundary structure, allowing for the dislocation to pass through the grain along a more favorable path. In this sense cross-slip can be seen as a mechanism for dislocations to overcome pinning sites such as grain boundary ledges and misfit regions, bringing an important new aspect to the subject of dislocation propagation within the nanocrystalline environment.

The authors acknowledge the financial support of European Commission (FP6-NANOMESO, Grant No. 016710) and thank the Swiss National Supercomputing Centre for the use of their computing facilities.

[1] J.P. Hirth and J. Lothe, Theory of Dislocations (John Wiley \& Sons, New York, 1982).

[2] W. Puschl, Prog. Mater. Sci. 47, 415 (2002).

[3] A. Seeger et al., Philos. Mag. 2, 323 (1957).

[4] G. Saada and P. Veyssiere, in Dislocations in Solids, edited by F.R.N. FNabarro and M.S. Duesbery (Elsevier Science, New York, 2002), p. 415.
[5] H. Van Swygenhoven, Science 296, 66 (2002).

[6] H. Van Swygenhoven and J. R. Weertman, Mater. Today 9 , 24 (2006).

[7] J. Schiotz, F. D. Di Tolla, and K. W. Jacobsen, Nature (London) 391, 561 (1998).

[8] J. Schiotz and K. W. Jacobsen, Science 301, 1357 (2003).

[9] A. G. Froseth, P. M. Derlet, and H. Van Swygenhoven, Acta Mater. 52, 5863 (2004).

[10] H. Van Swygenhoven, P. M. Derlet, and A. G. Froseth, Acta Mater. 54, 1975 (2006).

[11] H. Van Swygenhoven, M. Spaczer, and A. Caro, Acta Mater. 47, 3117 (1999).

[12] V. Yamakov et al., Nat. Mater. 3, 43 (2004).

[13] J. Schiotz et al., Phys. Rev. B 60, 11971 (1999).

[14] S. Brandstetter et al., Appl. Phys. Lett. 87, 231910 (2005).

[15] Z. Budrovic et al., Science 304, 273 (2004).

[16] G. Voronoi, J. Reine Angew. Math. 134, 198 (1908).

[17] P. M. Derlet and H. Van Swygenhoven, Phys. Rev. B 67, 014202 (2003).

[18] M. Parrinello and A. Rahman, J. Appl. Phys. 52, 7182 (1981).

[19] Y. Mishin et al., Mat. Res. Soc. Symp. Proc. (Materials Research Society, Pittsburgh, 1999), p. 533.

[20] H. Van Swygenhoven, P. M. Derlet, and A. G. Froseth, Nat. Mater. 3, 399 (2004).

[21] J. D. Honeycutt and H. C. Andersen, J. Phys. Chem. 91, 4950 (1987).

[22] J. Cormier, J. M. Rickman, and T. J. Delph, J. Appl. Phys. 89, 99 (2001).

[23] J. A.Zimmerman et al., Phys. Rev. Lett. 87, 165507 (2001).

[24] S. Brandstetter et al., Adv. Mater. 18, 1545 (2006).

[25] E. Bitzek et al. [Acta Mater. (to be published)].

[26] See, EPAPS Document No. E-PRLTAO-100-047824 for movies showing the temporal and spatial development of the cross-slipping dislocations within the nanocrystalline environment. For more information on EPAPS, see http:// www.aip.org/pubservs/epaps.html.

[27] N. Thompson, Proc. Phys. Soc. London Sect. B 66, 481 (1953).

[28] R. L. Fleischer, Acta Metall. 7, 134 (1959).

[29] J. Marian, J. Knap, and M. Ortiz, Acta Mater. 53, 2893 (2005).

[30] W. Cai et al., Dislocations in Solids (North-Holland, Amsterdam, 2004).

[31] T. Rasmussen et al., Phys. Rev. Lett. 79, 3676 (1997).

[32] T. Vegge et al., Phys. Rev. Lett. 85, 3866 (2000).

[33] H. Van Swygenhoven and A. Caro, Phys. Rev. B 58, 11246 (1998).

[34] H. Van Swygenhoven, P. M. Derlet, and A. Hasnaoui, Phys. Rev. B 66, 024101 (2002).

[35] V. Yamakov et al., Philos. Mag. Lett. 83, 385 (2003).

[36] D. Wolf et al., Z. Metallkd. 94, 1091 (2003).

[37] D. Wolf et al., Acta Mater. 53, 1 (2005).

[38] P. M. Derlet, Swygenhoven, and A. Hasnaoui, Philos. Mag. 83, 3569 (2003).

[39] P. M. Derlet, H. Van Swygenhoven, and A. Hasnaoui, Philos. Mag. 83, 3569 (2003).

[40] P. M. Derlet, S. Van Petegem, and H. Van Swygenhoven, Phys. Rev. B 71, 024114 (2005).

[41] S. Brandstetter, P. M. Derlet, S. Van Petegem, and H. Van Swygenhoven, Acta Mater. 56, 165 (2008). 